\title{
RESEARCH
}

Open Access

\section{Tau-related grey matter network breakdown across the Alzheimer's disease continuum}

Wiesje Pelkmans $s^{1,2^{*}}$ (D), Rik Ossenkoppele ${ }^{1,2}$, Ellen Dicks ${ }^{1}$, Olof Strandberg ${ }^{2}$, Frederik Barkhof ${ }^{3,4}$, Betty M. Tijms ${ }^{1}$, Joana B. Pereira ${ }^{2,5+}$ and Oskar Hansson ${ }^{2,6+}$

\begin{abstract}
Background: Changes in grey matter covariance networks have been reported in preclinical and clinical stages of Alzheimer's disease (AD) and have been associated with amyloid- $\beta$ (A $\beta$ ) deposition and cognitive decline. However, the role of tau pathology on grey matter networks remains unclear. Based on previously reported associations between tau pathology, synaptic density and brain structural measures, tau-related connectivity changes across different stages of AD might be expected. We aimed to assess the relationship between tau aggregation and grey matter network alterations across the AD continuum.
\end{abstract}

Methods: We included 533 individuals (178 Aß-negative cognitively unimpaired (CU) subjects, 105 Aß-positive CU subjects, 122 A $\beta$-positive patients with mild cognitive impairment, and 128 patients with AD dementia) from the BioFINDER-2 study. Single-subject grey matter networks were extracted from T1-weighted images and graph theory properties including degree, clustering coefficient, path length, and small world topology were calculated. Associations between tau positron emission tomography (PET) values and global and regional network measures were examined using linear regression models adjusted for age, sex, and total intracranial volume. Finally, we tested whether the association of tau pathology with cognitive performance was mediated by grey matter network disruptions.

Results: Across the whole sample, we found that higher tau load in the temporal meta-ROI was associated with significant changes in degree, clustering, path length, and small world values (all $p<0.001$ ), indicative of a less optimal network organisation. Already in CU A $\beta$-positive individuals associations between tau burden and lower clustering and path length were observed, whereas in advanced disease stages elevated tau pathology was progressively associated with more brain network abnormalities. Moreover, the association between higher tau load and lower cognitive performance was only partly mediated (9.3 to 9.5\%) through small world topology.

\footnotetext{
* Correspondence: w.pelkmans@amsterdamumc.nl

† Joana B. Pereira and Oskar Hansson contributed equally to this work.

${ }^{1}$ Alzheimer Center Amsterdam, Department of Neurology, Amsterdam

Neuroscience, Vrije Universiteit Amsterdam, Amsterdam UMC, Amsterdam,

The Netherlands

${ }^{2}$ Clinical Memory Research Unit, Department of Clinical Sciences, Lund

University, Malmö, Sweden

Full list of author information is available at the end of the article
}

(c) The Author(s). 2021 Open Access This article is licensed under a Creative Commons Attribution 4.0 International License, which permits use, sharing, adaptation, distribution and reproduction in any medium or format, as long as you give appropriate credit to the original author(s) and the source, provide a link to the Creative Commons licence, and indicate if changes were made. The images or other third party material in this article are included in the article's Creative Commons licence, unless indicated otherwise in a credit line to the material. If material is not included in the article's Creative Commons licence and your intended use is not permitted by statutory regulation or exceeds the permitted use, you will need to obtain permission directly from the copyright holder. To view a copy of this licence, visit http://creativecommons.org/licenses/by/4.0/ The Creative Commons Public Domain Dedication waiver (http://creativecommons.org/publicdomain/zero/1.0/) applies to the data made available in this article, unless otherwise stated in a credit line to the data. 
Conclusions: Our data suggest a close relationship between grey matter network disruptions and tau pathology in individuals with abnormal amyloid. This might reflect a reduced communication between neighbouring brain areas and an altered ability to integrate information from distributed brain regions with tau pathology, indicative of a more random network topology across different $A D$ stages.

Keywords: Alzheimer's disease, Graph theory, Grey matter network, MRI, PET, Tau

\section{Introduction}

Alzheimer's disease (AD) is generally thought to start with the aggregation of amyloid- $\beta(A \beta)$ in the brain, followed by deposition of neocortical tau pathology, synaptic dysfunction, atrophy, and cognitive decline [1-3]. However, the sequence and interactions of the pathophysiological processes and structural brain changes that occur during this long pre-dementia period are not well understood. Given that brain network abnormalities can already be observed in pre-dementia stages and contribute to cognitive impairment in $\mathrm{AD}$ [4-6], further clarification on the interrelations between brain connectivity with key pathological aggregates of $\mathrm{AD}$ could increase our understanding on the pathogenesis of $\mathrm{AD}$. One method to assess brain connectivity consists of measuring the similarity of cortical grey matter (GM) morphology, based on the notion that brain regions that engage in similar cognitive or behavioural processes tend to develop in a homologous way [7-9] through functional coactivation and/or axonal connectivity between brain regions [10-12]. Previous studies have shown GM network disruptions in preclinical AD [13-16], mild cognitive impairment [17-21], and AD dementia [5, 22-26]. Moreover, GM network disruptions have been related to increased $A \beta$ pathology $[13,15,16,27]$, while influences of tau pathology on GM brain networks remain unknown. Intraneuronal tau is thought to be more closely linked to synaptic function, brain integrity and clinical symptoms, than $A \beta$ plaques [28-31]. Therefore, we hypothesised that tau pathology may contribute to impaired network organisation in $\mathrm{AD}$. In this study, we tested whether tau deposition (measured with taupositron emission tomography [PET]) was associated with GM network alterations (measured with structural magnetic resonance imaging [MRI]) in individuals across the $\mathrm{AD}$ spectrum and whether these relationships were differentially linked with disease severity.

\section{Methods}

\section{Participants}

We included 533 individuals from the Swedish BioFINDER-2 study (NCT03174938) who underwent tauPET, structural MRI, and lumbar puncture to determine cerebrospinal fluid (CSF) $A \beta_{42} / A \beta_{40}$ levels as described previously [32]. In the present study, we included subjects $>50$ years of age with an abnormal CSF A $\beta$ status, resulting in three groups along the $A D$ continuum: $A \beta$ positive cognitively unimpaired $(\mathrm{CU})$ subjects (preclinical $\mathrm{AD}), \mathrm{A} \beta$-positive patients with mild cognitive impairment (prodromal $\mathrm{AD}$ ), and $\mathrm{A} \beta$-positive patients with $\mathrm{AD}$ dementia. Diagnosis was made according to clinical diagnostic criteria of the diagnostic and statistical manual of mental disorders (DSM)-5 [33]. In addition, an $A \beta$ negative cognitively unimpaired control group was included. All subjects underwent the Mini-Mental State Examination (MMSE) [34] and delayed word list recall test from the ADAS-Cog (Alzheimer's Disease Assessment Scale - Cognitive Subscale) [35] to assess global cognition and episodic memory, respectively. The inclusion and exclusion criteria of the BioFINDER-2 sub-cohorts are described in more detail in the eMethods section of the Supplementary material. All participants gave written informed consent to participate in the study. Ethical approval was given by the regional ethics committee at Lund University, Sweden. PET imaging procedures were approved by the Radiation protection committee at Skåne University Hospital and by the Swedish Medical Products Agency.

\section{MRI acquisition and pre-processing}

T1-weighted images were acquired using a magnetisationprepared rapid gradient echo sequence on a $3 \mathrm{~T}$ Siemens MAGNETOM Prisma scanner (Siemens Medical Solutions, Erlangen, Germany) using the following parameters: 178 slices, repetition time: $1950 \mathrm{~ms}$, echo time: $3.4 \mathrm{~ms}$, inversion time: $900 \mathrm{~ms}$, flip angle: $9^{\circ}, 1 \mathrm{~mm}$ isotropic voxels. All images were segmented into grey matter, white matter, and CSF using the Statistical Parametric Mapping (SPM12, https://www.fil.ion.ucl.ac.uk/spm/software/ spm12/) running in MATLAB (v2019b). The segmented grey matter images were resliced to $2 \times 2 \times 2 \mathrm{~mm}$ isotropic voxels to reduce the dimensionality of the data. Then, the images were parcellated into 100 anatomical brain regions using the Automated Anatomical Labelling (AAL3) atlas (all thalamic nuclei combined) [36], which was warped from standard space to subject space using subject-specific inverse normalisation parameters. The quality of these steps was visually assessed and two subjects had to be excluded due to misalignment of the brain atlas with the GM image. Total intracranial volume (TIV) was computed as the sum of grey matter, white matter, and CSF volumes. 


\section{Single-subject grey matter networks}

Single-subject grey matter networks were extracted from the native grey matter images using an automated template-free approach that has been previously published (https://github.com/bettytijms/Single_Subject_ Grey_Matter_Networks) [37]. For each subject, the grey matter network was built as a set of nodes connected by edges. The nodes were defined as cubes of $3 \times 3 \times 3$ voxels $\left(6 \times 6 \times 6 \mathrm{~mm}^{3}\right)$, which size was chosen based on 2 factors: (I) the minimum spatial resolution needed to still capture cortical folding has shown to be $3 \mathrm{~mm}$ [38], and (II) the practical computational limitations that exist with large matrices. These cubes keep the 3D structure of the cortex intact, and thereby contain information on grey matter intensity as well as spatial information between the voxels. In order to find the maximum correlation value with a target cube using Pearson correlation coefficients, each cube was rotated by an angle with multiples of $45^{\circ}$ over all axes, contributing to all positive connections. Self-connections were set to zero. The resulting similarity matrix containing all pairwise correlations was binarised using a threshold that reduced the chance of spurious correlations in the network to $5 \%$. This corresponded to a significance level of $p<0.05$ corrected for multiple comparisons using a permutationbased procedure [39]. The presence of an edge was indicated when the correlation between each pair of nodes exceeds this threshold. For regional network analyses, the corresponding atlas label for each cube was determined, this enabled averaging the GM network values and volume across nodes that were labelled according to that atlas region. A total of four subjects had to be excluded due to network calculation failure.

\section{Network properties}

Global and regional measures were calculated for each individual GM network. To assess global network properties the following measures were computed: network size (the total number of nodes in the network), degree (the number of edges), connectivity density (the ratio of existing connections to the maximum number of possible connections), clustering coefficient (the fraction of a node's neighbours that are also neighbours of each other), and path length (shortest path length between all pairs of nodes in the network). To normalise the network properties to random networks, we divided the average clustering coefficient and path length values by those values of five randomised reference networks of identical size and degree distribution, resulting in $\gamma$ and $\lambda$, respectively [40]. The ratio of $\gamma$ to $\lambda$ is defined as the small-world coefficient $\sigma$ [41], indicative of the optimal balance between information segregation and integration. To assess regional network properties, the degree, clustering coefficient, and path length were also calculated for each atlas brain area, i.e. region of interest (ROI). All network measures were computed with functions from the Brain Connectivity Toolbox (https://sites. google.com/site/bctnet/) [42], modified for large scale networks.

\section{PET acquisition and pre-processing}

Tau-PET imaging was conducted 70-90 min after injection of $365 \pm 20 \mathrm{MBq}\left[{ }^{18} \mathrm{~F}\right] \mathrm{RO} 948$ on digital GE Discovery MI scanners (General Electric Medical Systems) [43]. Low-dose CT scans were performed immediately prior to the PET scans for attenuation correction. PET data was reconstructed using VPFX-S (ordered subset expectation maximisation with time-of-flight and point spread function corrections) with 6 iterations and 17 subsets with $3 \mathrm{~mm}$ smoothing, standard $\mathrm{Z}$ filter, and $25.6-\mathrm{cm}$ field of view with a $256 \times 256$ matrix. After list-mode data was binned into $4 \times 5$-min time frames, PET images were motion corrected (rigid transformation using AFNI, 3dvolreg) [44], summed, and co-registered to their corresponding T1-weighted MR images. Standardised uptake value ratio (SUVR) images were created using the inferior cerebellar cortex as a reference region [32]. We calculated PET data both corrected and uncorrected for partial volume errors. Partial volume correction (PVC) was performed using the geometric transfer matrix method, as described in [45], PVC findings are available in the supplementary results section (eFigure 1, 2, eTable2).

To investigate the associations between tau pathology and network changes across different $\mathrm{AD}$ pathological stages, four composite regions were created based on the Braak staging scheme for neurofibrillary tangle pathology [46], adapted to PET space by [47]. These included the following brain regions as defined by the AAL atlas (see Supplementary eMethods 2 for details), and cover stage I-II (hippocampal formation), stage III-IV (fusiform, amygdala, cingulate and inferior and middle temporal cortices), and stage V-VI (widespread neocortical areas including the orbitofrontal, superior, middle and inferior frontal, precentral, paracentral, postcentral, precuneus, inferior and superior parietal, supramarginal, superior temporal, medial and lateral occipital cortices). In addition, a tau temporal meta-ROI capturing stages I to IV was calculated using the volume-weighted average of the corresponding regions [48].

\section{CSF collection and analysis}

CSF samples were obtained with a lumbar puncture and collected into $5 \mathrm{ml}$ LoBind polypropylene tubes handled according to the Alzheimer's Association Flow Chart for lumbar puncture [49]. Concentrations of $A \beta_{42}$ and $A \beta_{40}$ were quantified using enzyme-linked immunosorbent assays (ELISAs; INNOTEST, Fujirebio). Amyloid-status 
was determined using the $A \beta_{42} / A \beta_{40}$ ratio with a cutoff of $<0.089$ as defined abnormal in clinical practice at the Sahlgrenska University Hospital, Mölndal, Sweden [32].

\section{Statistical analyses}

Comparisons of demographical and clinical characteristics between groups were performed using chi-squared tests for categorical variables and one-way ANOVA for continuous variables.

To analyse the relationship between tau-PET (predictor) and global network measures (outcome), we used linear regression models adjusted for age, sex, and TIV (all models) and connectivity density (for path length, clustering, $\gamma, \lambda$, and $\sigma$ ), since higherorder measures have shown to depend on the number of nodes and edges in the network [50]. The association between tau-PET and global network measures was first tested across the whole sample, and then repeated within diagnostic groups with the interaction term group. Additionally, CSF $\mathrm{A} \beta_{42} / \mathrm{A} \beta_{40}$ was added to the linear regression model to test for an interaction effect CSF $A \beta_{42} / A \beta_{40}$ * tau on GM network changes. $Z$-scores for the network properties were calculated using the $A \beta$-negative control group mean and standard deviation, to aid comparisons of effect sizes between network properties. We further investigated the relationship of tau pathology with GM network disruptions at the local level across all AAL areas. Regional analyses were adjusted for age, sex,
TIV, local GM volume, and for clustering and path length also local degree. For global network analyses, we applied a threshold of $p<0.05$, corrected for multiple comparisons using the false discovery rate (FDR) correction method. For local network analyses, we adjusted for multiple testing using a Bonferroni correction $(p<0.05)$. Finally, we performed a mediation analysis using the Mediation package [51], to assess whether the association of tau pathology with cognitive performance was mediated by GM network disruptions. For these exploratory analyses, we assessed the small world coefficient only, as it indicates how randomly organised the network is, and it can be considered a summary measure of both $\gamma$ and $\lambda$. Analyses were performed using $\mathrm{R}$ (v4.0.2.) and visualised using Surf Ice (v2).

\section{Results \\ Participants}

In total, $178 \mathrm{~A} \beta$-negative $\mathrm{CU}$ controls, 105 preclinical $\mathrm{AD}, 122$ prodromal $\mathrm{AD}$, and $128 \mathrm{AD}$ dementia patients were included in the present study (mean age $=70.5 \pm$ 9.3 years; Table 1 ). Control subjects were younger and had a lower prevalence of apolipoprotein $\mathrm{E}(A P O E) \varepsilon 4$ than the other groups. As expected, lower MMSE scores, lower hippocampal volume, and higher tau-PET SUVr values were observed in the prodromal $\mathrm{AD}$ and $\mathrm{AD}$ dementia groups compared to the $\mathrm{CU}$ subjects.

Table 1 Subject characteristics
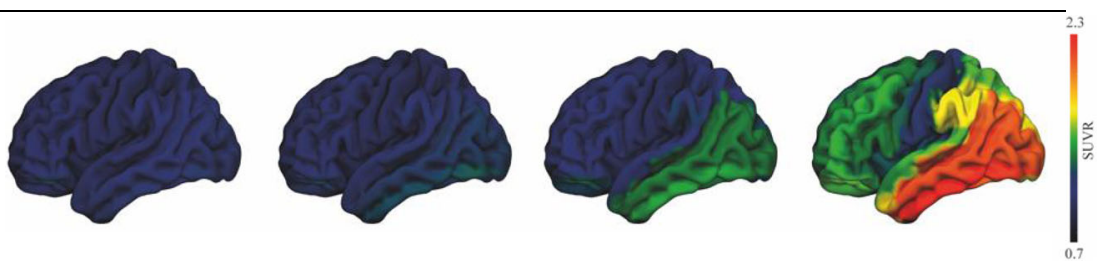

Control

$n=178$

Age, (y)

$66.1(10.3)^{\mathrm{b}, \mathrm{c}, \mathrm{d}}$

Preclinical AD

$n=105$

$103(58 \%)^{\mathrm{c}}$

$n=122$

Sex, $f(\%)$

$12.6(3.3)$

$29.1(1.1)^{\mathrm{c}, \mathrm{d}}$

$60(34 \%)^{b, c, d}$

APOEe4 carrier (\%)

Hippocampal volume $\left(\mathrm{cm}^{3}\right)$

$7.5(1.4)^{\mathrm{b}, \mathrm{c}, \mathrm{d}}$

$1.09(0.08)^{\mathrm{c}, \mathrm{d}}$ $71.8(9.3)^{\mathrm{a}}$

$56(53 \%)$

$12.3(3.5)$

$28.8(1.3)^{\mathrm{c}, \mathrm{d}}$

$65(62 \%)^{\mathrm{a}}$

$7.0(1.4)^{\mathrm{a}, \mathrm{c}, \mathrm{d}}$

$72.0(7.2)^{\mathrm{a}}$

$55(45 \%)^{\mathrm{a}}$

12.7 (4.5)

$27.0(1.9)^{\mathrm{a}, \mathrm{b}, \mathrm{d}}$

$83(69 \%)^{\mathrm{a}}$

$6.1(1.4)^{\mathrm{a}, \mathrm{b}, \mathrm{d}}$

$1.14(0.15)^{\mathrm{c}, \mathrm{d}}$
AD dementia

$n=128$

$74.1(6.8)^{\mathrm{a}}$

$71(55 \%)$

$12.1(4.4)$

$20.0(4.4)^{\mathrm{a}, \mathrm{b}, \mathrm{c}}$

$91(71 \%)^{\mathrm{a}}$

$5.0(1.1)^{\mathrm{a}, \mathrm{b}, \mathrm{c}, \mathrm{c}}$

$1.72(0.43)^{\mathrm{a}, \mathrm{b}, \mathrm{c}}$

Data are presented as mean $\pm \mathrm{SD}$, or $\mathrm{n}(\%) ; \mathrm{AD}$, Alzheimer's disease; F, female; MMSE, Mini Mental State Examination (0-30); ApoE, Apolipoprotein E; SUVR, Standardized uptake value ratio; ApoE 2 missing's; $a$ significantly different than control; $b$ significantly different than preclinical AD; $c$ significantly different than prodromal AD; $d$ significantly different than AD dementia at $p<0.05$; Surface plots display mean [18F]RO948 SUVR images. 
Table 2 Summary of global network measures

\begin{tabular}{|c|c|c|c|c|}
\hline & $\begin{array}{l}\text { Control } \\
n=178\end{array}$ & $\begin{array}{l}\text { Preclinical AD } \\
n=105\end{array}$ & $\begin{array}{l}\text { Prodromal AD } \\
n=122\end{array}$ & $\begin{array}{l}\text { AD dementia } \\
n=128\end{array}$ \\
\hline Network size & $7171.15(671.92)^{d}$ & $7007.46(672.18)^{d}$ & $6987.52(612.17)^{d}$ & $6666.01(581.66)^{a, b}$ \\
\hline Degree & $1193.11(148.21)^{d}$ & $1159.10(139.57)^{d}$ & $1140.61(133.61)^{d}$ & $1072.01(138.78)^{a, b}$ \\
\hline Connectivity density & $16.61(0.98)$ & $16.53(1.07)$ & $16.30(0.92)$ & $16.06(1.27)$ \\
\hline Clustering coefficient & $0.46(0.02)^{c, d}$ & $0.46(0.02)^{c, d}$ & $0.45(0.02)^{a, b, d}$ & $0.44(0.02)^{a, b, c}$ \\
\hline Path length & $1.98(0.03)^{c, d}$ & $1.97(0.03)^{c, d}$ & $1.96(0.03)^{a, b, d}$ & $1.96(0.03)^{a, b}, c$ \\
\hline Gamma & $1.58(0.11)^{c, d}$ & $1.54(0.12)^{c, d}$ & $1.50(0.09)^{a, b, d}$ & $1.43(0.09)^{a, b, c}$ \\
\hline Lambda & $1.08(0.01)^{c, d}$ & $1.08(0.02)^{c, d}$ & $1.07(0.01)^{a, b, d}$ & $1.06(0.01)^{a, b, c}$ \\
\hline Small world coefficient & $1.47(0.08)^{c, d}$ & $1.43(0.09)^{c, d}$ & $1.40(0.07)^{a, b, d}$ & $1.35(0.07)^{a, b, c}$ \\
\hline
\end{tabular}

Data are presented as mean \pm SD; $A D$ Alzheimer's disease; gamma is normalised clustering; lambda is normalised path length; ${ }^{a}$ significantly different than control; ${ }^{b}$ significantly different than preclinical AD; ' significantly different than prodromal AD; ${ }^{d}$ significantly different than AD dementia at $p<0.05$ when adjusting for age, sex, TIV, and connectivity density (clustering, path length, $\gamma, \lambda$, small world-only)

\section{Single-subject grey matter networks}

Networks had an average size of 6976 nodes (sd=664) and an average connectivity density of $16 \%(\mathrm{sd}=1)$ across all participants (Table 2). We observed that all grey matter network metrics showed lower values indicative of increased network abnormalities with advancing disease severity (Table 2). Differences between $A \beta$-negative controls and preclinical AD subjects were subtle and higherorder network differences lost significance after adjusting for covariates, including age. Compared to $A \beta$ negative controls, prodromal AD subjects showed lower clustering, path length, $\gamma, \lambda$, and small world coefficient values. Similar changes were observed in AD dementia, with in addition lower network size and degree compared to controls. In addition, all higher-order metrics were significantly lower in $\mathrm{AD}$ dementia compared to prodromal $\mathrm{AD}$ and significantly lower in prodromal $\mathrm{AD}$ compared to preclinical AD.

\section{Relationship between tau pathology and global grey matter network measures}

Across the whole sample, higher tau-PET values in the temporal meta-ROI were associated with lower values in all network properties (range $\beta=-0.25$ to $\beta=-1.12$; Fig. 1; Table 3), showing strongest associations for $\gamma$ and small world values. We observed similar relationships between GM network measures and tau signal in early (i.e. stage I/II) and late (i.e. stage V/VI) tau accumulation regions (Supplementary Results eTable 1).

When repeating the analyses stratified by diagnostic group, we found more significant associations between tau-PET and network measures with increasing disease

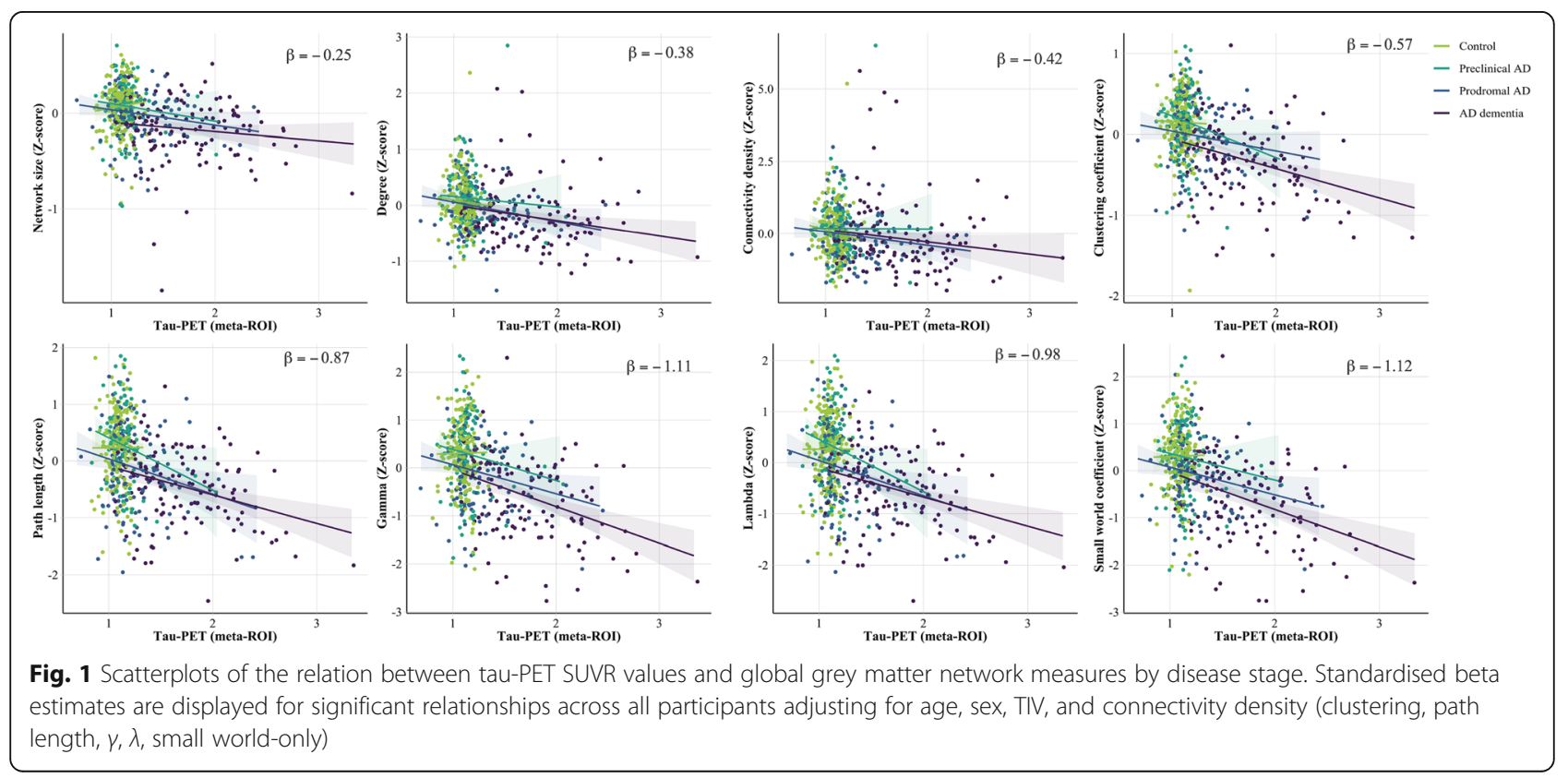


Table 3 Associations between global network measures and tau-PET

\begin{tabular}{|c|c|c|c|c|c|}
\hline Network property & Whole sample & Control & Preclinical AD & Prodromal AD & AD dementia \\
\hline Network size & $-0.25(0.03)^{* * *}$ & $0.14(0.26)$ & $-0.22(0.18)$ & $-0.17(0.10)$ & $-0.08(0.06)$ \\
\hline Degree & $-0.38(0.06)^{* * *}$ & $-0.37(0.46)$ & $-0.22(0.31)$ & $-0.36(0.17)$ & $-0.26(0.10)^{*}$ \\
\hline Connectivity density & $-0.42(0.13)^{* *}$ & $-1.03(0.97)$ & $-0.04(0.66)$ & $-0.48(0.35)$ & $-0.42(0.21)$ \\
\hline Clustering coefficient & $-0.57(0.05)^{* * *}$ & $-0.24(0.38)$ & $-0.58(0.26)^{*}$ & $-0.26(0.14)$ & $-0.34(0.08)^{* * *}$ \\
\hline Path length & $-0.87(0.08)^{* * *}$ & $-0.25(0.62)$ & $-1.04(0.42)^{*}$ & $-0.65(0.23)^{* *}$ & $-0.47(0.14)^{* *}$ \\
\hline Gamma & $-1.11(0.09)^{* * *}$ & $-0.23(0.71)$ & $-0.79(0.48)$ & $-0.65(0.25)^{*}$ & $-0.72(0.16)^{* * *}$ \\
\hline Lambda & $-0.98(0.09)^{* * *}$ & $-0.29(0.69)$ & $-1.15(0.47)^{*}$ & $-0.72(0.25)^{* *}$ & $-0.53(0.15)^{* *}$ \\
\hline Small world coefficient & $-0.12(0.10)^{* * *}$ & $-0.21(0.73)$ & $-0.70(0.49)$ & $-0.63(0.27)^{*}$ & $-0.75(0.16)^{* * *}$ \\
\hline
\end{tabular}

Data are presented as $\beta$ (SE); Network measures are Z trasformed; gamma is normalised clustering; lambda is normalised path length; SUVr values in temporal meta-ROl; Model is adjusted for age, sex, TIV, and connectivity density (clustering, path length, $\gamma$, $\lambda$, small world-only). ${ }^{*} p<0.05,{ }^{* *} p<0.01,{ }^{* * *} p<0.001$, FDR corrected.

severity: In preclinical $\mathrm{AD}$, higher tau-PET values in the meta-ROI were associated with lower clustering values $(\beta \pm \mathrm{SE} ;-0.58 \pm 0.26)$, path length $(-1.04 \pm 0.24)$, and $\lambda$ values ( $1.15 \pm 0.47$; all $p<.05$; Fig. 1 ; Table 3$)$. In prodromal $\mathrm{AD}$, higher tau-PET retention was related to lower path length (-0.65 \pm 0.23$), \gamma(-0.65 \pm 0.26), \lambda(-0.72 \pm 0.25)$, and small world coefficient values $(-0.63 \pm 0.27)$. In AD dementia, higher tau PET signal in the temporal metaROI was associated with lower degree $(-0.26 \pm 0.10)$, clustering $(-0.34 \pm 0.08)$, path length $(-0.47 \pm 0.14), \gamma(-0.72 \pm$ $0.16), \lambda(-0.53 \pm 0.15)$, and small world coefficient values $(-0.75 \pm 0.16)$. No association between tau-PET values and global network measures was observed in $\mathrm{A} \beta$-negative controls. Moreover, no group interaction effects were observed, indicating that the strength of the association between GM network measures and tau pathology did not differ significantly between disease stages.

When repeating the same analysis using the three ROIs specific for different tau stages, no association was observed between tau-PET values in the hippocampal formation (Braak I/II) and global network properties in preclinical AD participants (Supplementary Results eTable 1), suggesting that using the tau-PET signal in the temporal meta-ROI is more suitable in relation to network measures than early tau-accumulation regions. Tau pathology in limbic (Braak III-IV) and neocortical (Braak V-VI) areas correlated with decreased degree, clustering, path length, $\gamma, \lambda$, and small world coefficient values in prodromal and AD dementia subjects (Supplementary Results eTable 1). Moreover, we observed similar relationships between GM network measures and tau signal when repeating the analyses without the $A \beta$ negative control group (Supplementary Results eTable $3)$. Furthermore, there were no significant interactions between tau pathology and $A \beta_{42} / A \beta_{40}$ ratio values on GM network measures, suggesting that the associations between GM network alterations and tau pathology in $\mathrm{A} \beta$ positive individuals cannot be explained by CSF $A \beta$ levels (Supplementary Results eTable 3).

\section{Relationship between tau pathology and regional grey matter network measures}

Next, we examined the relationship between tau burden in the temporal meta-ROI with network measures at a local level in subjects on the AD continuum to assess a region-dependent effect. We observed different anatomical patterns of associations for each network measure. Lower clustering values were associated with higher tauPET SUVr values in widespread brain areas, showing the strongest associations in the precentral cortex, cingulate gyri, and frontal lobe (all $p_{\text {bonferroni }}<0.05$; Fig. 2). Associations between lower path length values and higher tau-PET retention were strongest in the cingulate gyri, precentral cortex, and inferior frontal cortex. Regions characterised by a lower degree showed also widespread associations with tau, which was most pronounced in the hippocampus, parahippocampus, amygdala, medial occipital cortex, and calcarine cortex (Fig. 2).

\section{Associations with cognitive performance}

We performed an exploratory analysis of the relationship of tau-pathology and small world topology with cognitive performance. Both higher tau-PET retention $(\beta \pm$ SE; $-8.6 \pm 0.37 ; 5.30 \pm 0.34$ ) and lower small world values $(\beta \pm$ SE; $2.14 \pm 0.19$; $-1.23 \pm 0.15)$ were significantly related to lower MMSE scores and more errors on the ADAS-Cog delayed word recall, respectively $(p<0.05$; Fig. 3). Moreover, small world values remained significant when controlling for tau-SUVr values. Mediation analysis revealed that lower MMSE scores and more recall errors associated with tau pathology were partially mediated by decreased small world values (9.3.0 to 9.5\%), but were mostly independent after controlling for the effect of small world coefficient.

\section{Discussion}

In a large well-phenotyped cohort, we observed that higher tau-PET retention is related to greater GM network disruptions in individuals across the $\mathrm{AD}$ continuum. 


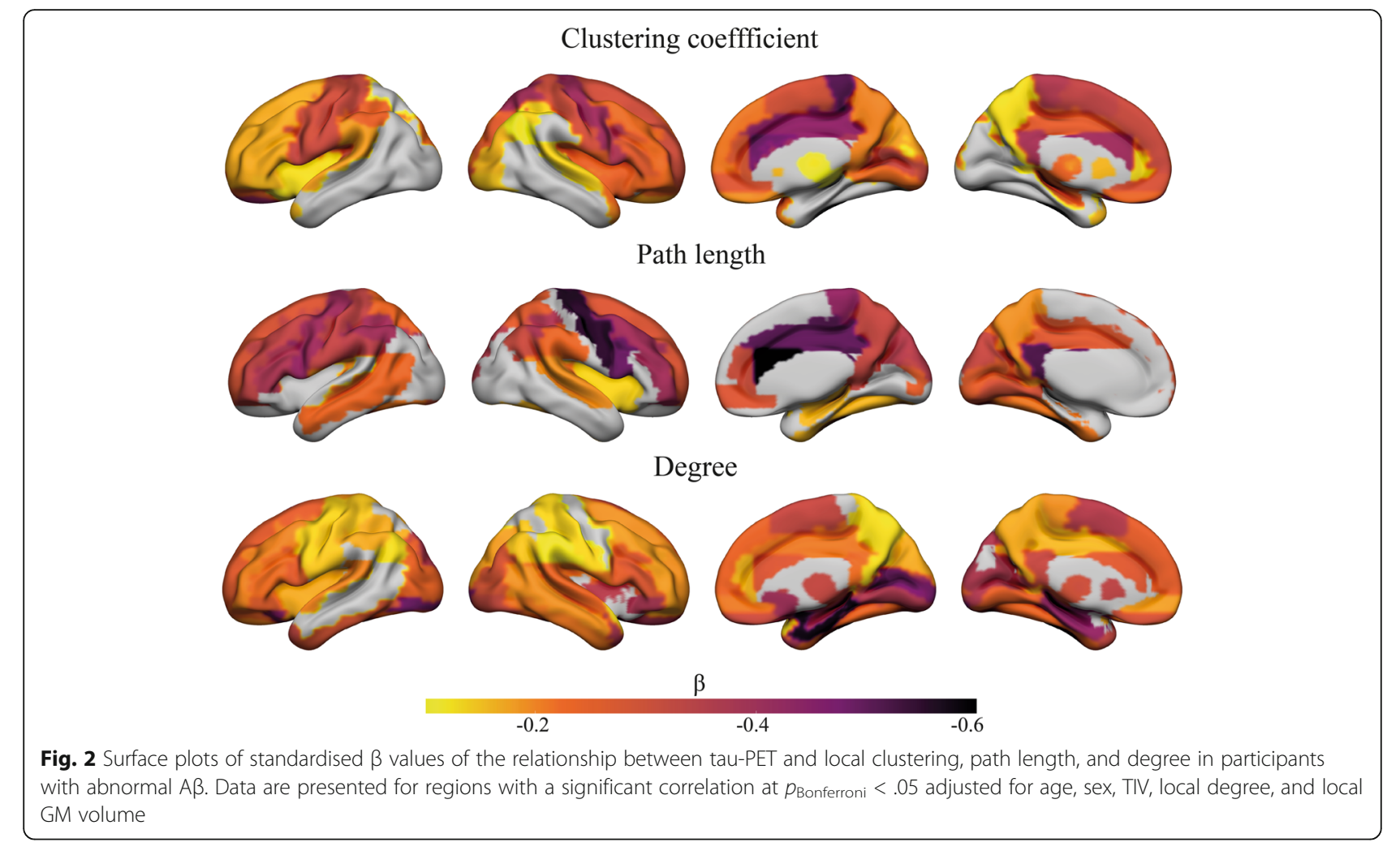

More advanced tau-related GM network abnormalities were observed with increasing disease severity. These findings suggest that tau pathology is associated with a reduced communication between neighbouring brain areas and an altered ability to integrate information from distributed brain regions indicative of a more random network topology across different AD stages.

Our results show a close relationship between GM network disruptions and tau pathology in individuals with abnormal amyloid. With increasing disease severity, we observed a greater tau load, as well as a greater number of abnormal GM network measures. As a result, the association of more network abnormalities with increased tau-PET retention may be largely based on differences in disease stage. Furthermore, when stratifying the analyses for diagnostic groups, we observed that distinct network measures were sensitive to different aspects and severity of neurodegeneration. Specifically, already in preclinical $\mathrm{AD}$ increased tau-PET signal was associated with network alterations showing lower clustering, lower path length values and normalised path length $\lambda$. These associations were absent in CU individuals with normal amyloid, indicating that the presence of $A \beta$ might significantly alter tau-related GM connectivity. These findings are in line with prior studies showing deterioration in network properties in preclinical AD $[13,14,52]$. In prodromal $\mathrm{AD}$ more network measures became abnormal with increasing tau pathology, additionally including changes in normalised clustering $\gamma$, and small world topology. In AD dementia patients nearly all network metrics were abnormal, suggesting that with pronounced neurodegeneration GM networks exhibit significant topological alterations, which is in line with previous studies in AD dementia [18, 22, 23]. Overall, these findings may indicate that a higher tau burden is associated with a higher dissimilarity (i.e. asynchronous atrophy) between neighbouring areas, and increased similarity between distant brain areas as a result of the progression of atrophy across the cortex, producing an increase in randomly connected nodes. Suggestive of an increasingly random network and reduction in small world organisation with disease progression [4, 42].

When characterising the spatial associations of tau pathology and GM network changes, tau deposition appeared to be widespread and strongly associated with lower clustering and path length in several regions of the default-mode network including the medial prefrontal cortex, precuneus, and anterior and posterior cingulate cortex. These findings may be related to early amyloid accumulating regions [53, 54]. We see that it is in these regions that clustering and path length further relates to tau, while also, more unexpectedly, in late tau accumulating regions such as the sensory-motor cortex and occipital lobe, strong associations of lower clustering and path length with tau were observed. Similar regional associations of GM network decline over time have been 

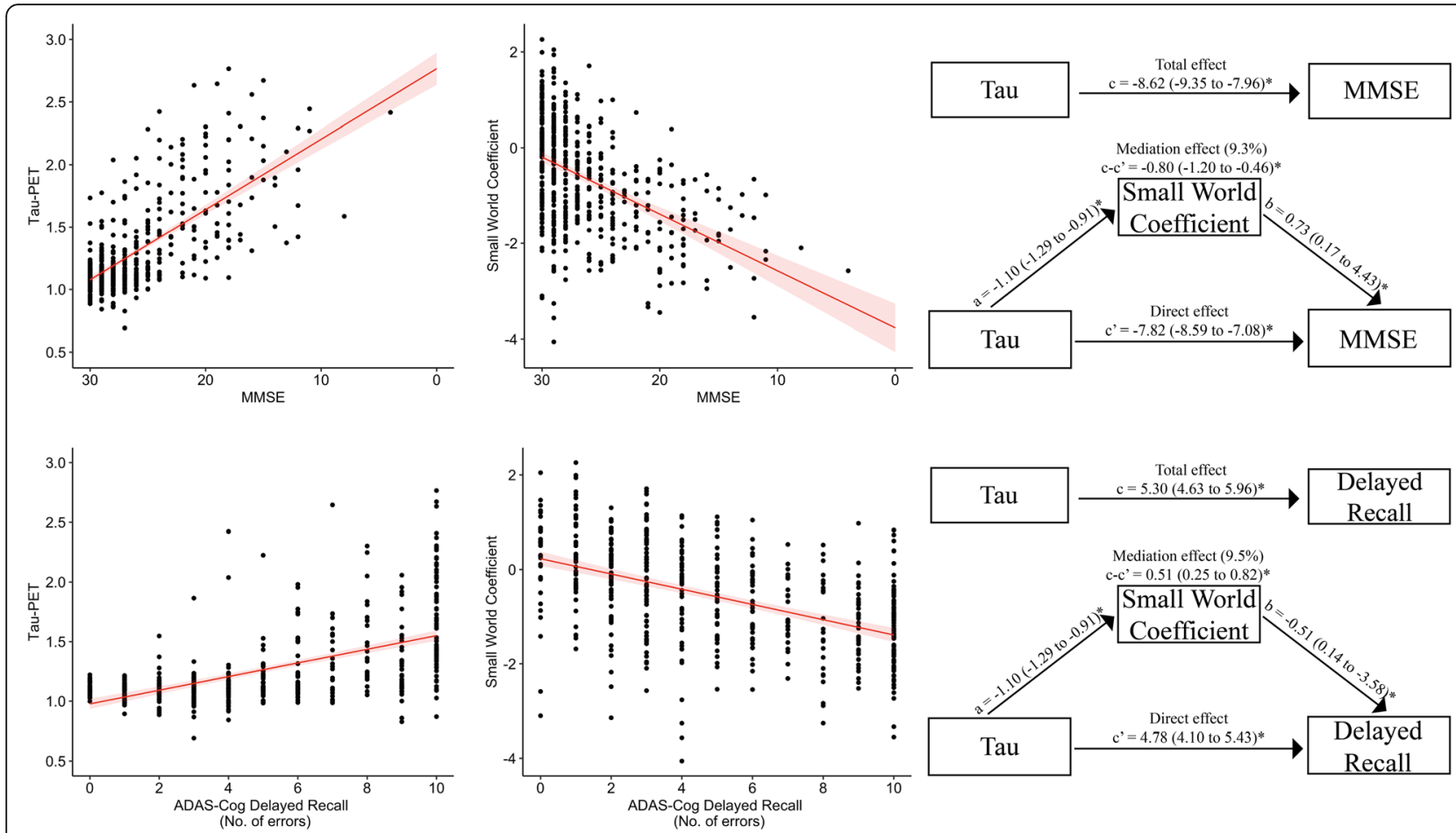

Fig. 3 MMSE and ADAS-Cog delayed recall performance in relation to Tau-PET and Small world coefficient. Scatterplot showing the association of tauPET SUVR values in the temporal meta-ROI and standardised grey matter network small world values with MMSE scores (top), and the ADAS-Cog delayed recall (bottom). Mediation analysis showing how small world topology mediates the effect of tau pathology on cognitive performance (right). Regression coefficients with a 95\% confidence interval are displayed. a The effect of tau load on small world topology. $\mathbf{b}$ The effect of small world topology on cognitive performance when controlling for tau. $\mathbf{c}$ The total effect of tau on cognition (without controlling for mediation effects). $\mathbf{c}^{\prime}$ The direct effect of tau pathology on cognition when adjusting for mediation. $\mathbf{c}-\mathbf{c}^{\prime}$, The mediation effect. ${ }^{*} p<0.05$

observed in individuals in presymptomatic stages of familial AD [55]. For lower degree, most strong correlations were observed in the medial temporal lobe, a region considered to be the tau epicentre where neurofibrillary tangles originate [46], suggestive of less connections in the medial temporal lobe with increasing tauPET signal.

Previous studies have related alterations in GM networks with abnormal amyloid aggregation in cognitively unimpaired individuals $[13,15,16,27]$, indicating that structural changes in GM networks are an early event in the pathophysiology of AD. Moreover, the presence of $\mathrm{A} \beta$ is hypothesised to increase the accumulation of tau outside of the medial temporal lobe [56-58], which may further accelerate network decline. As pathological tau shows reduced ability to stabilise microtubules, contributing to impaired axonal transport [30, 31], this may lead to further synaptic loss and neurodegeneration, resulting in substantial network damage and impaired cognition [28, 29, 59, 60]. As reflected by our work showing that both increased tau pathology and a more random GM network topology were associated with worse performance on a global cognition and episodic memory test. Both factors showed an independent contribution to worse cognitive performance, while mediation analyses also indicated that small world topology party mediated the effect of tau pathology on cognition.

\section{Limitations}

There are some limitations to our study. Firstly, this is a cross-sectional study that assumes three clinical stages of disease progression, and future longitudinal studies are needed to determine the temporal ordering in tau pathology and associated brain network changes more accurately. Secondly, for uniformity reasons, ROIs for both regional tau quantification and network topology calculation were created according to the AAL atlas. Unfortunately, the entorhinal cortex is not available as a separate region in this brain atlas; hence, in the current study stage I/II refers to the hippocampus and parahippocampus which includes the entorhinal cortex. This may have attenuated some of the results, but when testing the accuracy of the temporal meta-ROI in the AAL atlas with the Desikan-Killiany atlas, we observed a correlation of $R=.99$, rendering such effects likely to be minimal. Thirdly, since tau abnormalities are closely related to $A \beta$ pathology, it is difficult to know how specific the observed GM network alterations are to tau 
pathology. Strengths of our study include a large number of well-characterised participants. Moreover, the multimodal approach of combining structural MRI and tauPET imaging aids in understanding fundamental questions in the $\mathrm{AD}$ pathophysiology.

\section{Conclusions}

We found that GM network disruptions in $A D$ are strongly linked with tau burden, already in an early disease stage when cognition is within the normal range and becomes increasingly random with clinical progression. These findings provide more insight into the pathophysiological processes that contribute to brain network alterations in AD. An interesting future approach might lie in further investigating the prognostic value of GM single-subject networks in predicting cognitive decline and whether it can be implemented in clinical practice.

\section{Abbreviations}

AAL: Automated Anatomical Labelling; AD: Alzheimer's disease; AB: AmyloidB; APOE: Apolipoprotein E; CSF: Cerebrospinal fluid; CU: Cognitively unimpaired; DSM: Diagnostic and Statistical Manual of Mental Disorders; FDR: False discovery rate; GM: Grey matter; MMSE: Mini-Mental State Examination; MRI: Magnetic resonance imaging; PET: Positron emission tomography; PVC: Partial volume correction; ROI: Region of interest SPM: Statistical Parametric Mapping; SUVR: Standardised uptake value ratio; TIV: Total intracranial volume

\section{Supplementary Information}

The online version contains supplementary material available at https://doi. org/10.1186/s13195-021-00876-7

Additional file 1. Methods e1. Inclusion and exclusion criteria for the Swedish BioFINDER 2 study. Methods e2. AAL atlas ROls included in Braak staging. Results eTable 1. Results eTable 2. Results eTable 3. Results eFigure 1. Scatterplots of the relation between tau SUVr with PVC and global grey matter network measures by disease stage. Standardised beta estimates are displayed for significant relationships across all participants adjusting for age, sex, TIV, and connectivity density. Results eFigure 2. Surface plots of standardised $\beta$ values of the relationship between tau SUVr with PVC and local clustering, path length, and degree in participants with abnormal $A \beta$. Data are presented for regions with a significant correlation at $p_{\text {Bonferroni }}<.05$ adjusted for age, sex, TIV, local degree, and local GM volume.

\section{Acknowledgements}

The authors kindly thank all participants for their contribution.

\section{Authors' contributions}

WP, $\mathrm{RO}, \mathrm{JP}$, and $\mathrm{OH}$ contributed to the study concept and design. WP analysed the data. WP, RO, ED, OS, FB, BT, JP, and OH interpreted the data and revised the manuscript for intellectual content. All authors approved the final manuscript.

\section{Funding}

The BioFINDER study was supported by the Swedish Research Council (201600906), the Knut and Alice Wallenberg foundation (2017-0383), the Marianne and Marcus Wallenberg foundation (2015.0125), the Strategic Research Area MultiPark (Multidisciplinary Research in Parkinson's disease) at Lund University, the Swedish Alzheimer Foundation (AF-939932), the Swedish Brain Foundation (FO2019-0326), The Parkinson foundation of Sweden (1280/ 20), the Skåne University Hospital Foundation (2020-0000028), Regionalt Forskningsstöd (2020-0314) and the Swedish federal government under the
ALF agreement (2018-Projekt0279). The precursor of ${ }^{18} \mathrm{~F}$-RO948 was provided by Roche. WP is supported by a fellowship grant (WE.15-2019-14) by Alzheimer Nederland. ED is supported by Race Against Dementia (https:// www.raceagainstdementia.com/). FB is supported by the NIHR biomedical research centre at UCLH. JBP is supported by the Swedish Research Council, Alzheimerfonden, Stratneuro, Center for Medical Innovation, Stohnes and Gamla Tjänarinnor. The funding sources had no role in the design and conduct of the study; in the collection, analysis, interpretation of the data; or in the preparation, review, or approval of the manuscript. Open Access funding provided by Lund University.

\section{Availability of data and materials}

Anonymised study data for the primary analyses presented in this report are available on request from any qualified investigator for purposes of replicating the results.

\section{Declarations}

Ethics approval and consent to participate

All participants gave written informed consent to participate in the study. Ethical approval was given by the regional ethics committee at Lund University, Sweden. PET imaging procedures were approved by the Radiation protection committee at Skåne University Hospital and by the Swedish Medical Products Agency.

\section{Consent for publication}

Not applicable.

\section{Competing interests}

$\mathrm{RO}$ is an associate editor at Alzheimer's Research \& Therapy. FB is a consultant to Biogen Idec, Merck-Serono, Novartis, Roche, IXICO Ltd, GeNeuro, and Combinostics. He received grants for Amyloid imaging for the Prevention of Alzheimer Disease (Innovative Medicines Initiative), European Progression Of Neurological Disease (H2020), UK Multiple Sclerosis Society, Dutch Multiple Sclerosis Society, National Institute Health Research University College London Biomedical Research Centre. OH has acquired research support (for the institution) from AVID Radiopharmaceuticals, Biogen, Eli Lilly, Eisai, GE Healthcare, Pfizer, and Roche. In the past 2 years, he has received consultancy/speaker fees from AC Immune, Alzpath, Biogen, Cerveau and Roche.

\section{Author details}

${ }^{1}$ Alzheimer Center Amsterdam, Department of Neurology, Amsterdam Neuroscience, Vrije Universiteit Amsterdam, Amsterdam UMC, Amsterdam, The Netherlands. ${ }^{2}$ Clinical Memory Research Unit, Department of Clinical Sciences, Lund University, Malmö, Sweden. ${ }^{3}$ Department of Radiology \& Nuclear Medicine, Amsterdam Neuroscience, Vrije Universiteit Amsterdam, Amsterdam UMC, Amsterdam, The Netherlands. ${ }^{4}$ Queen Square Institute of Neurology and Centre for Medical Image Computing, University College London, London, UK. ${ }^{5}$ Division of Clinical Geriatrics, Department of Neurobiology, Care Sciences and Society, Karolinska Institute, Stockholm, Sweden. ${ }^{6}$ Memory Clinic, Skåne University Hospital, Malmö, Sweden.

Received: 2 March 2021 Accepted: 9 July 2021

Published online: 13 August 2021

\section{References}

1. Jack CR, Bennett DA, Blennow K, et al. NIA-AA Research Framework: Toward a biological definition of Alzheimer's disease. Alzheimers Dementia. 2018; 14(4):535-62. https://doi.org/10.1016/j.jalz.2018.02.018.

2. Selkoe DJ, Hardy J. The amyloid hypothesis of Alzheimer's disease at 25 years. EMBO Molecular Medicine. 2016;8(6):595-608. https://doi.org/10.152 52/emmm.201606210.

3. Scheltens $P$, de Strooper B, Kivipelto $M$, Holstege $H$, Chételat $G$, Teunissen CE, et al. Alzheimer's disease. Lancet. 2021;6736:1-13.

4. van den Heuvel MP, Sporns O. A cross-disorder connectome landscape of brain dysconnectivity. Nat Rev Neurosci. 2019;20(7):435-46. https://doi.org/1 0.1038/s41583-019-0177-6.

5. Seeley WW, Crawford RK, Zhou J, Miller BL, Greicius MD, Michael D. Neurodegenerative Diseases Target Large-Scale Human Brain Networks. Neuron. 2009;62(1):42-52. https://doi.org/10.1016/j.neuron.2009.03.024. 
6. Jones DT, Knopman DS, Gunter JL, Graff-Radford J, Vemuri P, Boeve BF, et al. Cascading network failure across the Alzheimer's disease spectrum. Brain. 2016;139(2):547-62. https://doi.org/10.1093/brain/awv338.

7. Alexander-Bloch A, Giedd JN, Bullmore E. Imaging structural co-variance between human brain regions. Nat Rev Neurosci. 2013;14(5):322-36. https:// doi.org/10.1038/nrn3465.

8. Evans AC. Networks of anatomical covariance. Neurolmage. 2013;80:489504. https://doi.org/10.1016/j.neuroimage.2013.05.054.

9. Mechelli A. Structural Covariance in the Human Cortex. J Neurosci. 2005; 25(36):8303-10. https://doi.org/10.1523/JNEUROSCl.0357-05.2005.

10. Alexander-Bloch A, Raznahan A, Bullmore E, Giedd J. The Convergence of Maturational Change and Structural Covariance in Human Cortical Networks. J Neurosci. 2013;33(7):2889-99. https://doi.org/10.1523/ JNEUROSCI.3554-12.2013.

11. Gong G, He Y, Chen ZJ, Evans AC. Convergence and divergence of thickness correlations with diffusion connections across the human cerebral cortex. Neurolmage. 2012;59(2):1239-48. https://doi.org/10.1016/j.neuroima ge.2011.08.017.

12. Doucet GE, Moser DA, Rodrigue A, Bassett DS, Glahn DC, Frangou S. PersonBased Brain Morphometric Similarity is Heritable and Correlates With Biological Features. Cereb Cortex. 2019;29(2):852-62. https://doi.org/10.1093/ cercor/bhy287.

13. ten Kate M, Visser PJ, Bakardjian H, et al. Gray Matter Network Disruptions and Regional Amyloid Beta in Cognitively Normal Adults. Front Aging Neurosci. 2018;10:1-11.

14. Verfaillie SCJ, Slot RER, Dicks E, Prins ND, Overbeek JM, Teunissen CE, et al. A more randomly organized grey matter network is associated with deteriorating language and global cognition in individuals with subjective cognitive decline. Hum Brain Mapp. 2018;39(8):3143-51. https://doi.org/10.1 002/hbm.24065.

15. Voevodskaya O, Pereira JB, Volpe G, Lindberg O, Stomrud E, van Westen D, et al. Altered structural network organization in cognitively normal individuals with amyloid pathology. Neurobiol Aging. 2018;64:15-24. https:// doi.org/10.1016/j.neurobiolaging.2017.11.014.

16. Tijms BM, ten Kate M, Wink AM, et al. Gray matter network disruptions and amyloid beta in cognitively normal adults. Neurobiol Aging. 2016;37:154-60. https://doi.org/10.1016/j.neurobiolaging.2015.10.015.

17. Dicks E, Tijms BM, ten Kate M, Gouw AA, Benedictus MR, Teunissen CE, et al. Gray matter network measures are associated with cognitive decline in mild cognitive impairment. Neurobiol Aging. 2018;61:198-206. https://doi.org/1 0.1016/j.neurobiolaging.2017.09.029.

18. Pereira JB, Mijalkov M, Kakaei E, Mecocci P, Vellas B, Tsolaki M, et al. Disrupted Network Topology in Patients with Stable and Progressive Mild Cognitive Impairment and Alzheimer's Disease. Cereb Cortex. 2016;26(8): 3476-93. https://doi.org/10.1093/cercor/bhw128.

19. Yao Z, Zhang Y, Lin L, Zhou Y, Xu C, Jiang T. Abnormal cortical networks in mild cognitive impairment and alzheimer's disease. PLoS Comput Biol. 2010; 6(11):e1001006. https://doi.org/10.1371/journal.pcbi.1001006.

20. Montembeault M, Rouleau I, Provost J-S, Brambati SM. Altered Gray Matter Structural Covariance Networks in Early Stages of Alzheimer's Disease. Cereb Cortex. 2016;26(6):2650-62. https://doi.org/10.1093/cercor/bhv105.

21. Spreng RN, Turner GR. Structural Covariance of the Default Network in Healthy and Pathological Aging. J Neurosci. 2013;33(38):15226-34. https:// doi.org/10.1523/JNEUROSCI.2261-13.2013.

22. He Y, Chen Z, Evans A. Structural Insights into Aberrant Topological Patterns of Large-Scale Cortical Networks in Alzheimer's Disease. J Neurosci. 2008; 28(18):4756-66. https://doi.org/10.1523/JNEUROSCI.0141-08.2008.

23. Tijms BM, Möller C, Vrenken H, Wink AM, de Haan W, van der Flier WM, et al. Single-Subject Grey Matter Graphs in Alzheimer's Disease. PLoS One. 2013;8(3):e58921. https://doi.org/10.1371/journal.pone.0058921.

24. Li Y, Wang Y, Wu G, Shi F, Zhou L, Lin W, et al. Discriminant analysis of longitudinal cortical thickness changes in Alzheimer's disease using dynamic and network features. Neurobiol Aging. 2012;33:427.e15-30.

25. John M, Ikuta T, Ferbinteanu J. Graph analysis of structural brain networks in Alzheimer's disease: beyond small world properties. Brain Struct Funct. 2017; 222(2):923-42. https://doi.org/10.1007/s00429-016-1255-4.

26. Kim H-J, Shin J-H, Han CE, Kim HJ, Na DL, Seo SW, et al. Using Individualized Brain Network for Analyzing Structural Covariance of the Cerebral Cortex in Alzheimer's Patients. Front Neurosci. 2016;10:1-11.

27. Oh H, Mormino EC, Madison C, Hayenga A, Smiljic A, Jagust WJ. $\beta-$ Amyloid affects frontal and posterior brain networks in normal aging.
Neurolmage. 2011;54(3):1887-95. https://doi.org/10.1016/j.neuroimage.2 010.10 .027 .

28. Ossenkoppele R, Schonhaut DR, Schöll M, Lockhart SN, Ayakta N, Baker SL, et al. Tau PET patterns mirror clinical and neuroanatomical variability in Alzheimer's disease. Brain. 2016;139(5):1551-67. https://doi.org/10.1093/bra in/aww027.

29. Nelson PT, Alafuzoff I, Bigio EH, Bouras C, Braak H, Cairns NJ, et al. Correlation of Alzheimer Disease Neuropathologic Changes With Cognitive Status: A Review of the Literature. J Neuropathol Exp Neurol. 2012;71(5): 362-81. https://doi.org/10.1097/NEN.0b013e31825018f7.

30. Menkes-Caspi N, Yamin HG, Kellner V, Spires-Jones TL, Cohen D, Stern EA. Pathological Tau Disrupts Ongoing Network Activity. Neuron. 2015;85(5): 959-66. https://doi.org/10.1016/j.neuron.2015.01.025.

31. Coomans EM, Schoonhoven DN, Tuncel H, Verfaillie SCJ, Wolters EE, Boellaard R, et al. In vivo tau pathology is associated with synaptic loss and altered synaptic function. Alzheimers Res Ther. 2021;13(1):35. https://doi. org/10.1186/s13195-021-00772-0.

32. Leuzy A, Smith R, Ossenkoppele R, Santillo A, Borroni E, Klein G, et al. Diagnostic Performance of RO948 F 18 Tau Positron Emission Tomography in the Differentiation of Alzheimer Disease From Other Neurodegenerative Disorders. JAMA Neurol. 2020;77(8):955. https://doi.org/10.1001/jamaneurol.2 020.0989.

33. American Psychiatric Association. Diagnostic and Statistical Manual of Mental Disorders. Arlington. 2013. https://doi.org/10.1176/appi.books. 9780890425596

34. Folstein MF, Robins LN, Helzer JE. The Mini-Mental State Examination. Arch Gen Psychiatry. 1983;40(7):812. https://doi.org/10.1001/archpsyc.1983.01 790060110016.

35. Rosen WG, Mohs RC, Davis KL. A new rating scale for Alzheimer's disease. Am J Psychiatry. 1984;141:1356-64.

36. Rolls ET, Huang C-C, Lin C-P, Feng J, Joliot M. Automated anatomical labelling atlas 3. Neurolmage. 2020;206:116189. https://doi.org/10.1016/j. neuroimage.2019.116189.

37. Tijms BM, Seris $P$, Willshaw DJ, Lawrie SM. Similarity-based extraction of individual networks from gray matter MRI scans. Cereb Cortex. 2012;22(7): 1530-41. https://doi.org/10.1093/cercor/bhr221.

38. Kiselev VG, Hahn KR, Auer DP. Is the brain cortex a fractal? Neurolmage. 2003:20(3):1765-74. https://doi.org/10.1016/S1053-8119(03)00380-X.

39. Noble WS. How does multiple testing correction work? Nat Biotechnol. 2009:27(12):1135-7. https://doi.org/10.1038/nbt1209-1135.

40. Maslov S, Sneppen K. Specificity and Stability in Topology of Protein Networks. Science. 2002;296(5569):910-3. https://doi.org/10.1126/science.1 065103.

41. Humphries MD, Gurney K. Network 'Small-World-Ness': A Quantitative Method for Determining Canonical Network Equivalence. PLoS One. 2008; 3(4):e0002051. https://doi.org/10.1371/journal.pone.0002051.

42. Bullmore E, Sporns O. Complex brain networks : graph theoretical analysis of structural and functional systems. Nat Rev Neurol. 2009;10(3):186-98. https://doi.org/10.1038/nrn2575.

43. Yap SY, Frias B, Wren MC, Schöll M, Fox NC, Årstad E, et al. Discriminatory ability of next-generation tau PET tracers for Alzheimer's disease. Brain. 2021 https://doi.org/10.1093/brain/awab120.

44. Cox J.S. RW; H (1996) AFNI: Software for analysis and visualization of functional magnetic resonance neuroimages. Comput Biomed Res 29:162173, 3, DOl: https://doi.org/10.1006/cbmr.1996.0014

45. Rousset OG, Ma Y, Evans AC. Correction for partial volume effects in PET: principle and validation. J Nucl Med. 1998:39(5):904-11.

46. Braak H, Braak E. Neuropathological stageing of Alzheimer-related changes. Acta Neuropathol. 1991;82(4):239-59. https://doi.org/10.1007/ BF00308809.

47. Cho H, Choi JY, Hwang MS, Kim YJ, Lee HM, Lee HS, et al. In vivo cortical spreading pattern of tau and amyloid in the Alzheimer disease spectrum. Ann Neurol. 2016;80(2):247-58. https://doi.org/10.1002/ana.24711.

48. Ossenkoppele R, Rabinovici GD, Smith R, Cho H, Schöll M, Strandberg $\mathrm{O}$, et al. Discriminative accuracy of [18F]flortaucipir positron emission tomography for Alzheimer disease vs other neurodegenerative disorders. JAMA. 2018;320(11):1151-62. https://doi.org/10.1001/jama.201 8.12917.

49. Blennow K, Hampel H, Weiner M, Zetterberg H. Cerebrospinal fluid and plasma biomarkers in Alzheimer disease. Nat Rev Neurol. 2010;6(3):131-44. https://doi.org/10.1038/nrneurol.2010.4 
50. van Wijk BCM, Stam CJ, Daffertshofer A. Comparing Brain Networks of Different Size and Connectivity Density Using Graph Theory. PLoS One. 2010;5(10):e13701. https://doi.org/10.1371/journal.pone.0013701.

51. Tingley D, Yamamoto T, Hirose K, Keele L, Imai K. mediation : R Package for Causal Mediation Analysis. J Stat Softw. 2014:59:1-38.

52. Dicks $E$, Vermunt $L$, van der Flier WM, Barkhof F, Scheltens P, Tijms BM. Grey matter network trajectories across the Alzheimer's disease continuum and relation to cognition. Brain Commun. 2020;2:1-15.

53. Buckner RL, Snyder AZ, Shannon BJ, LaRossa G, Sachs R, Fotenos AF, et al. Molecular, Structural, and Functional Characterization of Alzheimer's Disease: Evidence for a Relationship between Default Activity, Amyloid, and Memory. J Neurosci. 2005;25(34):7709-17. https://doi.org/10.1523/JNEUROSCI.217705.2005 .

54. Palmqvist S, Schöll M, Strandberg O, Mattsson N, Stomrud E, Zetterberg H, et al. Earliest accumulation of $\beta$-amyloid occurs within the default-mode network and concurrently affects brain connectivity. Nat Commun. 2017; 8(1):1214. https://doi.org/10.1038/s41467-017-01150-x.

55. Vermunt L, Dicks E, Wang G, et al. Single-subject grey matter network trajectories over the disease course of autosomal dominant Alzheimer's disease. Brain Commun. 2020;2:1-14.

56. Price JL, Morris JC. Tangles and Plaques in Nondemented Aging and "Preclinical" Alzheimer's Disease. Ann Neurol. 1999:45(3):358-68. https://doi. org/10.1002/1531-8249(199903)45:3<358::AID-ANA12>3.0.CO;2-X.

57. seok BM, Cho H, Lee HS, Choi JY, Lee JH, Ryu YH, et al. Temporal trajectories of in vivo tau and amyloid- $\beta$ accumulation in Alzheimer's disease. Eur J Nucl Med Mol Imaging. 2020;47(12):2879-86. https://doi.org/10.1007/s00259-02004773-3.

58. Jack $C R$, Wiste $H J$, Botha $H$, et al. The bivariate distribution of amyloid- $\beta$ and tau: relationship with established neurocognitive clinical syndromes. Brain. 2019;142(10):3230-42. https://doi.org/10.1093/brain/awz268.

59. Pereira JB, Janelidze $S$, Ossenkoppele $R$, et al. Untangling the association of amyloid- $\beta$ and tau with synaptic and axonal loss in Alzheimer's disease. Brain. 2021;144:310-24. https://doi.org/10.1093/brain/awaa395.

60. Schultz AP, Chhatwal JP, Hedden T, Mormino EC, Hanseeuw BJ, Sepulcre J, et al. Phases of Hyperconnectivity and Hypoconnectivity in the Default Mode and Salience Networks Track with Amyloid and Tau in Clinically Normal Individuals. J Neurosci. 2017;37(16):4323-31. https://doi.org/10.1523/ JNEUROSCI.3263-16.2017.

\section{Publisher's Note}

Springer Nature remains neutral with regard to jurisdictional claims in published maps and institutional affiliations.

Ready to submit your research? Choose BMC and benefit from:

- fast, convenient online submission

- thorough peer review by experienced researchers in your field

- rapid publication on acceptance

- support for research data, including large and complex data types

- gold Open Access which fosters wider collaboration and increased citations

- maximum visibility for your research: over $100 \mathrm{M}$ website views per year

At $\mathrm{BMC}$, research is always in progress.

Learn more biomedcentral.com/submissions 